\title{
Comparative evaluation of different pretreatment methods on biogas production from paddy straw
}

\author{
Vishwas Garg $^{1 *}$, Rouf Ahmad Dar ${ }^{1}$ and Urmila Gupta Phutela ${ }^{2}$ \\ ${ }^{1}$ Department of Microbiology, Punjab Agricultural University, Ludhiana-141004 (Punjab), INDIA \\ ${ }^{2}$ School of Renewable Energy Engineering, Punjab Agricultural University, Ludhiana-141004 (Punjab), INDIA \\ *Corresponding author. E-mail: garg.vishwas11@gmail.com
}

Received: December 2, 2016; Revised received: March 29, 2017; Accepted: July 29, 2017

\begin{abstract}
The present investigations observed the effect of chemical, enzymatic, biological and microwave pretreatment on paddy straw for enhancement of biogas production. Chopped and soaked paddy straw was subjected to chemicals $\mathrm{Na}_{2} \mathrm{CO}_{3}(1 \%)$ and $\mathrm{NaOH}(2 \%)$ concentrations, microwave irradiation (720 watt, $30 \mathrm{~min}$ ), fungal (spawn impregnated, 7 days) and crude silicase (24 hrs) pretreatment. The proximate and chemical analysis showed $16.0 \%$ and $12.1 \%$ reduction in lignin and silica content in the case of $\mathrm{Na}_{2} \mathrm{CO}_{3}$ pretreated paddy straw whereas $23.0 \%$ and $46.8 \%$ reduction was observed in enzymatic pretreatment with $43.7 \%$ and $31.1 \%$ enhancement in biogas production respectively. This clearly indicates that Pleurotus ostreatus MTCC 142 is silicolytic as well as ligninolytic in nature. Enzymatic pretreatment was also compared with, microwave (30 min) and fungal pretreatment which showed $31.2 \%$ and $32.8 \%$ reduction in silica content enhancing biogas production by $19.7 \%$ and $42.6 \%$ respectively. $\mathrm{NaOH}$ pretreatment showed a maximum increase in biogas production i.e. $49.7 \%$ as compared to $1 \%$ pretreated sample which showed $28.5 \%$ enhancement. The results indicated that the $\mathrm{NaOH}$ pretreatment was one of the potential methods to increase biogas production of paddy straw.
\end{abstract}

Keywords: Biogas production, Ligninolytic, Pleurotus ostreatus, Pretreatment, Silicolytic

\section{INTRODUCTION}

Rice, one of the most staple food, provides nutrition and calorie intake to nearly half of the world's population (Arvanitoyannis et al., 2008). Every ton of rice harvesting produces 0.8 tons of straw (595.92 million tonnes 2014) which stands in the field as waste biomass but with high energy potential (FAO Rice and Market monitor, 2014). According to the global status energy report approximately $147 \mathrm{GW}$ power from biomass was generated at the end of 2015 and over 8.1 million jobs were estimated to be increased in relation to the renewable energy sector (www.ren21.net). Rice straw possesses various uses such as paper making, resin binders and also as livestock feed but the higher silica and lower phosphorus content makes it a poor feedstock for animals (http://www.carrb.com/84rpt/ Straw Uses. htm), therefore burning of straw is a common practice mainly in the harvest season which emits about $144719 \mathrm{mg}$ of total particulate matter from burning of rice straw in the field (Gadde et al., 2009). A total of $3 \mathrm{~kg}$ particulate matter, $60 \mathrm{~kg} \mathrm{CO}, 1460 \mathrm{~kg}$ $\mathrm{CO}_{2}, 199 \mathrm{~kg}$ ash and $2 \mathrm{~kg} \mathrm{\textrm {SO } _ { 2 }}$ is produced via open burning of one ton rice straw, which not only reduces soil productivity but substantially contributes to air pollution (Streets et al., 2003 and Akhter et al., 2015). Rice straw is one of the most abundant lignocellulosic biomass in the world (Saini and Saini, 2015). It is mainly composed of (32-47\%) cellulose and (19-27\%) hemicelluloses that can be readily hydrolysed into fermentable sugars (Saha, 2003 and Raeisi et al., 2016) via of anaerobic digestion, a series of biological processes which involves the conversion of organic material into biogas and digestate in the presence of anaerobic microorganisms and in the absence of oxygen (Kangle et al., 2012). The rate and extent of degradation are the two important parameters that affect the anaerobic digestion process. High biodegradation rate reduces the reactor size and makes it economically attractive (Contreras et al., 2012). High amount of organic acids such as lactic acid, butyric acids and various amino acids have also been produced using rice straw as a substrate as described by Younas et al., (2016) who produced 58.1\% lactic acid from $1 \mathrm{M}$ $\mathrm{NaOH}$ pretreated rice straw while $2.6 \mathrm{~g} /(\mathrm{L} . \mathrm{d})$ butyric acid was produced from paddy straw via consolidate bioprocessing (Ai et al., 2016).

The major challenge is to digest the multilayer structure of rice straw shielded by the presence of lignin (Jafri et al., 2011 and Werle et al., 2013). Therefore 
effective methods are required to degrade the straw at higher rate for maximum biogas production. Over the past decades a large number of chemical (acid, alkali, oxidizing agents, and organic solvents), physicochemical (steam explosion, ammonia fiber explosion), physical (comminution, milling and grinding), biological, or a combination of these pretreatment methods had been investigated on variety of feedstock (Kumar et al., 2009) which have enhanced biogas production at a higher rate. As the chemicals used are not economically cheap and also provokes a hazardous threat towards the environment, therefore biological methods have gained researcher's attention in the current era. A wide range of bacteria and fungi are known to solubilize insoluble silicates by producing minerals, organic acids and a variety of chelating agents (Henderson and Duff, 1965). Fungi such as Pleurotus ostreatus, Pleurotus florida, Phanerochaete chrysosporium, Cyathusstercoreus, Ceriporiopsis subvermispora, were reported to elevate the digestibility of paddy straw (Blanchette et al., 1992, Chen et al., 1996, Gammal et al., 1998 and Taniguchi et al., 2005), however Phanerochaete and Pleurotus showed beneficial effects, degrading lignin by $40 \%$ and $60 \%$ in cotton straw (Platt et al., 1984). The present study was aimed to find out maximum paddy straw degradation and biogas yield by various pretretment methods.

\section{MATERIALS AND METHODS}

Substrate and chemicals: The paddy straw was procured from the research field of Punjab Agricultural University, Ludhiana after harvesting of crop and was chopped $(3-5 \mathrm{~cm})$ with a chopping machine for its easy handling, which was later stored in polythene bags at room temperature. Cattle dung was procured from dairy farm of Guru Angad Dev Veterinary and Animal Sciences University (GADVASU) Ludhiana. Digested cattle dung slurry was obtained from a nearby working biogas plant in the School of Renewable Energy and Engineering, PAU Ludhiana.

All the chemicals used for proximate and chemical analysis, media and solutions preparation were of analytical grade and were purchased from Hi-Media, SRL, Sigma and S D fine chemicals Pvt. Ltd.

Silicase production and extraction: Silica peptone broth was inoculated with P.ostreatus MTCC 142 culture and incubated at $28^{\circ} \mathrm{C} \pm 2^{\circ} \mathrm{C}$ at $200 \mathrm{rpm}$. After 0,2 , 4, 6 and 8 days of incubation the broth was filtered and the filtrate was then centrifuged at $15000 \mathrm{rpm}$ for a period of $15 \mathrm{~min}$ in a cooling centrifuge (Remi Instruments Ltd.). The clear supernatant was used as crude enzyme extract. The silicase activity in the crude enzyme extract was determined by the method described by Toender and Borchert, (2014).

Pretreatment of paddy straw

Chemical pretreatment of paddy straw: Two chemicals sodium carbonate $\left(\mathrm{Na}_{2} \mathrm{CO}_{3}\right)$ and sodium hydrox- ide $(\mathrm{NaOH})$ of concentrations $1 \%$ and $2 \%$ each were used. Two hundred and fifty gram of chopped and soaked paddy straw was suspended in these chemical solutions for $24 \mathrm{hrs}$ at room temperature. After the desired period of soaking, the solution was decanted off and the paddy straw was washed under tap water till the washings were clean, colourless and neutral to $\mathrm{pH}$ paper. A small amount as a sample was taken and dried overnight in a laboratory oven at $70^{\circ} \mathrm{C}-80^{\circ} \mathrm{C}$. After drying the sample was ground in order to get uniform and reproducible results. Otherwise, the paddy straw formed clumps on pretreatment and was not fully accessible to different chemicals/solutions used in proximate analysis. The samples were stored in polythene bags for proximate and chemical analysis and the rest straw was fed to the digesters for biogas production.

Microwave pretreatment of paddy straw: A domestic microwave oven MC-2681DS produced by LG Electronics India Pvt. Ltd was used in this study for microwave pretreatment. The microwave frequency was $2550 \mathrm{MHz}$ and the power was set at $720 \mathrm{~W}$. Two hundred and fifty gram of chopped and soaked paddy straw was suspended in $500 \mathrm{ml}$ of water in an autoclavable bag. The bags were pierced with pins to avoid excess pressure inside the bag and placed at the centre of microwave oven on the circular plate for its pretreatment for $30 \mathrm{~min}$. The bags were allowed to cool and required sample was washed and dried for proximate and chemical analysis whereas rest straw was fed to the reactors for biogas production.

\section{Biological pretreatment of paddy straw}

Preparation of inoculum: The inoculum needed for pretreatment of paddy straw was prepared on wheat grains. The grains were washed under tap and boiled for 25-35 min until tender. The surplus amount of water was decanted off. The grains were then mixed with $4 \%$ calcium carbonate $\left(\mathrm{CaCO}_{3}\right)$ and $2 \%$ gypsum $\left(\mathrm{CaSO}_{4}\right)$ which were later dispensed into empty glucose bottles $(250 \mathrm{~g} /$ bottle) and were autoclaved for 90 minutes. After cooling the bottles were inoculated by placing two $5 \mathrm{~mm}$ bits of the freshly prepared culture of P.ostreatus MTCC 142 each on opposite side of the bottle. The bottles were incubated at $28^{\circ} \mathrm{C} \pm 2^{\circ} \mathrm{C}$, till mycelium completely impregnates the wheat grains (710 days).

Mixing of inoculum with paddy straw: The chopped paddy straw $(250 \mathrm{~g})$ was soaked in water overnight. The excess water was drained off and the paddy straw was then incorporated with the inoculum (fungus impregnated wheat grains) at the rate of $10 \% \mathrm{w} / \mathrm{w}$ ratio ( $25 \mathrm{~g}$ in $250 \mathrm{~g}$ paddy straw). The bags were allowed to incubate at room temperature for 1 week. After completion of the incubation period, the samples of pretreated paddy straw were taken and dried at $70^{\circ} \mathrm{C}$ in an oven. The paddy straw was grounded to powder and stored in polythene bags which were later used for 
determining the change in proximate and chemical composition of paddy straw.

\section{Enzymatic hydrolysis}

Silicase pretreatment: Silicase was produced from P.ostreatus MTCC 142 under optimal conditions the crude enzyme extract in different concentrations i.e. $15 \mathrm{U} / 10 \mathrm{~g}, 30 \mathrm{U} / 10 \mathrm{~g}$ and $45 \mathrm{U} / 10 \mathrm{~g}$ was used to pretreat the paddy straw. Two hundred and fifty gram of chopped (3-5 cm size) and soaked paddy straw was mixed with crude enzyme extract with different concentrations separately and was allowed to react for a period of $24 \mathrm{hrs}$ at room temperature. The samples were dried in an oven at $80^{\circ} \mathrm{C}$ overnight, grinded to powder and were stored in polythene bags for determining the variation in proximate and chemical analysis. The samples for proximate and chemical analysis were studied and the remaining straw were fed to the digesters for biogas production.

Solid state anaerobic digestion setup for biogas production (SSAD): Chopped and soaked paddy straw pretreated with different methods chemical, microwave, biological and enzymatic respectively was subjected to biogas production. The untreated (control) and pretreated paddy straw were digested in batch anaerobic digesters. The batch system composed of a $2 \mathrm{~L}$ glass reactor, 2L glass bottle for gas collection which was filled with dilute hydrochloric acid and solutions ( $\mathrm{pH}<3$ to avoid $\mathrm{CO}_{2}$ dissolution) and $1 \mathrm{~L}$ liquid collection beaker. Biogas production was measured by water displacement method i.e. by measuring the amount of water displaced by the gas produced in a millilitre. Eight groups (each of control, chemical, microwave, biological and enzymatic pretreated paddy straw) each containing triplicate anaerobic digesters were set up at $35^{\circ} \mathrm{C} \pm 2^{\circ} \mathrm{C}$. The digesters were mixed with $50 \mathrm{~g}$ cattle dung (acting as inducer) and $25 \mathrm{ml}$ cattle dung slurry (acting as inoculums). Reactors were then carefully examined for any leaks to inspect close tight rubber stopper and screw caps. The samples were taken both at the initial stage and after a period of 45 days for proximate and chemical analysis. Biogas production for each reactor was measured every day and level of water was maintained in gas collecting chamber.

Analytical methods: Standard methods of AOAC (2000) were followed for the determination of Total solids, Volatile solids, Total organic carbon, ash, cellulose, hemi-cellulose, lignin and silica content.

Statistical analysis: The experimental data which was generated from the experiments was analysed using the SAS system. The data was analysed by Tukey's HSD method for multiple comparisons. Standard deviation was also calculated for the data.

\section{RESULTS AND DISCUSSION}

Effect of different pretreatment methods on paddy straw digestibility and biogas production

$\mathrm{Na}_{2} \mathrm{CO}_{3}$ pretreatment: Pretreatment of rice straw by $\mathrm{Na}_{2} \mathrm{CO}_{3}$ was applied at room temperature to enhance the degradation of rice straw. Table 1 presents the hydrolytic behaviour of $\mathrm{Na}_{2} \mathrm{CO}_{3}$ pretreatment on paddy straw digestibility with maximum total solids, volatile solids and total organic carbon reduction by $2.2 \%$, $8.9 \%$ and $8.9 \%$ in $2 \%$ pretreated sample respectively. On the contrary ash content was increased by $34.7 \%$ which can be due to the decrease in volatile solid content. Maximum reduction in cellulose by $10.5 \%$ and

Table 1. Change in proximate and chemical composition of $\mathrm{Na}_{2} \mathrm{CO}_{3}$ and $\mathrm{NaOH}$ pretreated paddy straw.

\begin{tabular}{|c|c|c|c|c|c|c|c|c|c|c|}
\hline \multirow{3}{*}{$\begin{array}{l}\text { Composi- } \\
\text { tion of PS } \\
\%\end{array}$} & \multirow{2}{*}{\multicolumn{2}{|c|}{ Control }} & \multicolumn{4}{|c|}{$\mathrm{Na}_{2} \mathrm{CO}_{3}$ concentration $(\%)$} & \multicolumn{4}{|c|}{$\mathrm{NaOH}$ concentration $(\%)$} \\
\hline & & & \multicolumn{2}{|c|}{1} & \multicolumn{2}{|c|}{2} & \multicolumn{2}{|c|}{1} & \multicolumn{2}{|c|}{2} \\
\hline & Initial & Final & Initial & Final & Initial & Final & Initial & Final & Initial & Final \\
\hline $\begin{array}{l}\text { Total } \\
\text { solids }\end{array}$ & $96.2 \pm 0.4^{\mathrm{a}}$ & $\begin{array}{c}95.4 \pm 0.7^{\mathrm{b}} \\
(0.8 \downarrow)\end{array}$ & $96.0 \pm 0.8^{\mathrm{a}}$ & $\begin{array}{c}94.8 \pm 0.4^{\mathrm{d}} \\
(1.25 \downarrow)\end{array}$ & $94.2 \pm 0.9^{\mathrm{e}}$ & $\begin{array}{c}92.1 \pm 1.0^{\mathrm{d}} \\
(2.2 \downarrow)\end{array}$ & $96.1 \pm 0.6^{\mathrm{a}}$ & $\begin{array}{c}95.0 \pm 0.2^{\mathrm{b}} \\
(1.1 \downarrow)\end{array}$ & $95.7 \pm 0.4^{b}$ & $\begin{array}{c}94.0 \pm 0.6^{\mathrm{e}} \\
(1.7 \downarrow)\end{array}$ \\
\hline $\begin{array}{l}\text { Volatile } \\
\text { solids }\end{array}$ & $86.8 \pm 0.5^{\mathrm{a}}$ & $\begin{array}{c}85.5 \pm 0.3^{\mathrm{b}} \\
(1.4 \downarrow)\end{array}$ & $84.4 \pm 0.2^{\mathrm{c}}$ & $\begin{array}{c}78.5 \pm 0.7^{\mathrm{e}} \\
(6.9 \downarrow)\end{array}$ & $82.6 \pm 0.7^{\mathrm{d}}$ & $\begin{array}{c}75.2 \pm 0.8^{\mathrm{f}} \\
(8.9 \downarrow)\end{array}$ & $82.6 \pm 0.2^{\mathrm{d}}$ & $\begin{array}{c}75.5 \pm 0.15^{\mathrm{f}} \\
(8.5 \downarrow)\end{array}$ & $83.8 \pm 0.6^{\mathrm{c}}$ & $\begin{array}{c}71.2 \pm 0.1^{g} \\
(15.0 \downarrow)\end{array}$ \\
\hline $\begin{array}{l}\text { Total } \\
\text { organic } \\
\text { carbon }\end{array}$ & $48.2 \pm 0.4^{\mathrm{a}}$ & $\begin{array}{c}47.5 \pm 0.7^{\mathrm{a}} \\
(1.4 \downarrow)\end{array}$ & $46.8 \pm 1.1^{\mathrm{b}}$ & $\begin{array}{c}43.6 \pm 0.5^{\mathrm{d}} \\
(6.8 \downarrow)\end{array}$ & $45.8 \pm 1.2^{\mathrm{c}}$ & $\begin{array}{c}41.7 \pm 0.5^{\mathrm{e}} \\
(8.8 \downarrow)\end{array}$ & $45.8 \pm 0.8^{\mathrm{c}}$ & $\begin{array}{c}41.9 \pm 0.6^{\mathrm{e}} \\
(8.5 \downarrow)\end{array}$ & $46.5 \pm 0.2^{\mathrm{b}}$ & $\begin{array}{c}39.5 \pm 0.2^{\mathrm{f}} \\
(15.1 \downarrow)\end{array}$ \\
\hline Ash & $13.2 \pm 0.6^{\mathrm{h}}$ & $\begin{array}{c}14.5 \pm 0.6^{\mathrm{h}} \\
(9.8 \uparrow)\end{array}$ & $15.6 \pm 0.6^{\mathrm{g}}$ & $\begin{array}{c}22.5 \pm 0.2^{\mathrm{c}} \\
(44.2 \uparrow)\end{array}$ & $18.4 \pm 0.4^{\mathrm{e}}$ & $\begin{array}{c}24.8 \pm 0.6^{\mathrm{b}} \\
(34.7 \uparrow)\end{array}$ & $17.4 \pm 0.7^{\mathrm{f}}$ & $\begin{array}{c}24.5 \pm 0.3^{\mathrm{b}} \\
(40.8 \uparrow)\end{array}$ & $19.8 \pm 0.6^{\mathrm{d}}$ & $\begin{array}{c}28.8 \pm 0.4^{\mathrm{a}} \\
(45.4 \uparrow)\end{array}$ \\
\hline Cellulose & $46.2 \pm 0.5^{\mathrm{b}}$ & $\begin{array}{c}45.8 \pm 0.2^{\mathrm{b}} \\
(0.8 \downarrow)\end{array}$ & $44.0 \pm 0.8^{\mathrm{c}}$ & $\begin{array}{c}40.1 \pm 0.6^{\mathrm{e}} \\
(8.8 \downarrow)\end{array}$ & $42.6 \pm 0.9^{\mathrm{d}}$ & $\begin{array}{c}38.1 \pm 0.8^{\mathrm{g}} \\
(10.5 \downarrow)\end{array}$ & $45.0 \pm 0.6^{\mathrm{a}}$ & $\begin{array}{c}39.4 \pm 0.2^{\mathrm{f}} \\
(12.4 \downarrow)\end{array}$ & $44.8 \pm 0.9^{\mathrm{a}}$ & $\begin{array}{c}36.9 \pm 0.3^{\mathrm{h}} \\
(17.6 \downarrow)\end{array}$ \\
\hline $\begin{array}{l}\text { Hemicel- } \\
\text { lulose }\end{array}$ & $25.0 \pm 0.3^{\mathrm{a}}$ & $\begin{array}{c}24.6 \pm 0.1^{\mathrm{b}} \\
(1.6 \downarrow)\end{array}$ & $24.2 \pm 0.5^{\mathrm{b}}$ & $\begin{array}{c}22.6 \pm 0.4^{\mathrm{d}} \\
(6.6 \downarrow)\end{array}$ & $24.4 \pm 0.9^{b}$ & $\begin{array}{c}21.5 \pm 0.2^{\mathrm{e}} \\
(11.8 \downarrow)\end{array}$ & $26.4 \pm 0.7^{\mathrm{a}}$ & $\begin{array}{c}23.6 \pm 0.4^{\mathrm{c}} \\
(10.6 \downarrow)\end{array}$ & $26.2 \pm 0.4^{\mathrm{a}}$ & $\begin{array}{c}20.1 \pm 0.2^{f} \\
(23.2 \downarrow)\end{array}$ \\
\hline Lignin & $6.5 \pm 0.2^{\mathrm{a}}$ & $\begin{array}{c}6.2 \pm 0.2^{\mathrm{a}} \\
(4.6 \downarrow)\end{array}$ & $5.8 \pm 0.09^{\mathrm{b}}$ & $\begin{array}{c}5.5 \pm 0.1^{\mathrm{b}} \\
(5.1 \downarrow)\end{array}$ & $5.6 \pm 0.04^{\mathrm{b}}$ & $\begin{array}{l}4.7 \pm 0.1^{\mathrm{c}} \\
(16.0 \downarrow)\end{array}$ & $6.2 \pm 0.4^{\mathrm{a}}$ & $\begin{array}{c}5.7 \pm 0.3^{\mathrm{b}} \\
(8.0 \downarrow)\end{array}$ & $5.5 \pm 0.6^{\mathrm{b}}$ & $\begin{array}{c}4.3 \pm 0.1^{\mathrm{c}} \\
(21.8 \downarrow)\end{array}$ \\
\hline Silica & $7.9 \pm 0.1^{\mathrm{a}}$ & $\begin{array}{c}7.7 \pm 0.1^{\mathrm{a}} \\
(2.5 \downarrow)\end{array}$ & $7.6 \pm 0.07^{\mathrm{a}}$ & $\begin{array}{c}7.0 \pm 0.2^{\mathrm{b}} \\
(7.8 \downarrow)\end{array}$ & $7.4 \pm 0.09^{\mathrm{a}}$ & $\begin{array}{c}6.5 \pm 0.09^{\mathrm{c}} \\
(12.1 \downarrow)\end{array}$ & $6.2 \pm 0.1^{\mathrm{c}}$ & $\begin{array}{l}7.0 \pm 0.7^{\mathrm{b}} \\
(12.9 \uparrow)\end{array}$ & $5.0 \pm 0.09^{\mathrm{d}}$ & $\begin{array}{l}6.0 \pm 0.2^{\mathrm{c}} \\
(20.0 \uparrow)\end{array}$ \\
\hline
\end{tabular}

*Control: Untreated paddy straw; PS: Paddy straw; values are means \pm standard deviation $(n=3)$; Initial indicate sample taken after pretreatment; Final indicate sample taken after 45 days of pretreatment; value in parenthesis indicates \% increase $(\uparrow)$; \% decrease $(\downarrow)$ w.r.t initial value; Within the same row, different small letters indicate a significant difference between parameters under the different pretreatments based on Tukey's HSD test $(\mathrm{p}<0.05)$ 
Table 2.Silicase production in submerged fermentation.

\begin{tabular}{lcc}
\hline Incubation period (days) & Silicase activity (U/ml) & Carbonic anhydrase activity (U/ml) \\
\hline 0 & $0.046 \pm 0.09$ & $0.05 \pm 0.01$ \\
2 & $0.45 \pm 0.06$ & $0.52 \pm 0.02$ \\
4 & $0.67 \pm 0.04$ & $0.70 \pm 0.02$ \\
6 & $0.85 \pm 0.03$ & $0.87 \pm 0.03$ \\
8 & $1.05 \pm 0.06$ & $1.10 \pm 0.02$ \\
10 & $0.73 \pm 0.04$ & $0.76 \pm 0.01$ \\
\hline
\end{tabular}

*Experimental conditions; Medium used silica peptone broth; Incubation temperature: $30^{\circ} \mathrm{C} \pm 2{ }^{\circ} \mathrm{C}$; $\mathrm{pH} 6.5$; mycelial bit size 5 $\mathrm{mm}$; values are means \pm standard deviation $(\mathrm{n}=3)$

Table 3. Change in proximate and chemical composition of paddy straw by pretreatment with crude silicase enzyme

\begin{tabular}{lllll}
\hline Parameters (\%) & Control & $\mathbf{1 5}$ U/10 g PS & 30 U/10 g PS & 45 U/10 g PS \\
\hline Total solids & $96.2 \pm 0.4^{\mathrm{c}}$ & $96.7 \pm 0.5^{\mathrm{c}}(0.5 \uparrow)$ & $97.3 \pm 0.7^{\mathrm{b}}(1.1 \uparrow)$ & $98.0 \pm 0.4^{\mathrm{a}}(1.8 \uparrow)$ \\
Volatile solids & $86.8 \pm 0.5^{\mathrm{a}}$ & $84.5 \pm 0.5^{\mathrm{b}}(2.6 \downarrow)$ & $82.6^{\mathrm{c}} \pm 0.1^{\mathrm{c}}(4.8 \downarrow)$ & $81.0 \pm 0.6^{\mathrm{d}}(6.6 \downarrow)$ \\
Total organic carbon & $48.2 \pm 0.4^{\mathrm{a}}$ & $46.9 \pm 1.0^{\mathrm{b}}(2.7 \downarrow)$ & $45.8 \pm 0.8^{\mathrm{c}}(4.9 \downarrow)$ & $45.0 \pm 0.3^{\mathrm{d}}(6.6 \downarrow)$ \\
Ash & $13.2 \pm 0.6^{\mathrm{d}}$ & $15.5 \pm 0.7^{\mathrm{c}}\left(17.4^{\mathrm{a}} \uparrow\right)$ & $17.4 \pm 0.6^{\mathrm{b}}(31.8 \uparrow)$ & $19.0 \pm 0.5^{\mathrm{a}}\left(43.9^{\mathrm{a}} \uparrow\right)$ \\
Cellulose & $46.2 \pm 0.5^{\mathrm{a}}$ & $44.2 \pm 0.6^{\mathrm{b}}(4.3 \downarrow)$ & $41.1 \pm 0.6^{\mathrm{c}}(11.0 \downarrow)$ & $38.4 \pm 0.8^{\mathrm{d}}(16.8 \downarrow)$ \\
Hemicellulose & $25.0 \pm 0.3^{\mathrm{d}}$ & $27.6 \pm 0.5^{\mathrm{c}}(10.4 \uparrow)$ & $29.6 \pm 0.8^{\mathrm{b}}(18.4 \uparrow)$ & $31.9 \pm 0.6^{\mathrm{a}}(27.6 \uparrow)$ \\
Lignin & $6.5 \pm 0.2^{\mathrm{a}}$ & $6.2 \pm 0.5^{\mathrm{a}}(4.6 \uparrow)$ & $5.8 \pm 0.8^{\mathrm{b}}(10.7 \downarrow)$ & $5.0 \pm 0.4^{\mathrm{c}}(23.0 \downarrow)$ \\
Silica & $7.9 \pm 0.1^{\mathrm{a}}$ & $7.0 \pm 1.1^{\mathrm{b}}(11.3 \downarrow)$ & $6.5 \pm 0.4^{\mathrm{c}}(17.7 \downarrow)$ & $4.2 \pm 0.2^{\mathrm{d}}(46.8 \downarrow)$ \\
\hline
\end{tabular}

*Experimental conditions; soaking period $24 \mathrm{hrs;} \mathrm{values} \mathrm{are} \mathrm{means} \pm$ standard deviation $(\mathrm{n}=3)$; value in parenthesis indicates $\%$ increase $(\uparrow)$; \% decrease $(\downarrow)$ w.r.t initial value; Within the same row, different small letters indicate a significant difference between parameters under the different pretreatments based on Tukey's HSD test $(p<0.05)$

Table 4. Change in proximate and chemical composition of silicase pretreated paddy straw after biogas production

\begin{tabular}{lcccc}
\hline \multirow{2}{*}{ Parameters } & \multicolumn{2}{c}{ Control } & \multicolumn{2}{c}{ Pretreated straw (45 units/10g PS) } \\
\cline { 2 - 5 } & Initial & Final & Initial & Final \\
\hline Total solids & $96.2 \pm 0.4^{\mathrm{c}}$ & $95.4 \pm 0.7^{\mathrm{d}}(0.8 \downarrow)$ & $98.0 \pm 0.4^{\mathrm{b}}$ & $98.6 \pm 0.4^{\mathrm{a}}(0.6 \uparrow)$ \\
Volatile solids & $86.8 \pm 0.5^{\mathrm{a}}$ & $85.5 \pm 0.3^{\mathrm{b}}(1.4 \downarrow)$ & $81.0 \pm 0.6^{\mathrm{c}}$ & $77.2 \pm 0.4^{\mathrm{d}}(4.6 \downarrow)$ \\
Total organic carbon & $48.2 \pm 0.4^{\mathrm{a}}$ & $47.5 \pm 0.7^{\mathrm{b}}(1.4 \downarrow)$ & $45.0 \pm 0.3^{\mathrm{c}}$ & $42.8 \pm 0.2^{\mathrm{d}}(4.8 \downarrow)$ \\
Ash & $13.2 \pm 0.6^{\mathrm{d}}$ & $14.5 \pm 0.6^{\mathrm{c}}(9.8 \uparrow)$ & $19.0 \pm 0.5^{\mathrm{b}}$ & $22.8 \pm 0.1^{\mathrm{a}}(20.0 \uparrow)$ \\
Cellulose & $46.2 \pm 0.5^{\mathrm{a}}$ & $45.8 \pm 0.2^{\mathrm{b}}(0.8 \downarrow)$ & $38.4 \pm 0.8^{\mathrm{c}}$ & $34.5 \pm 0.3^{\mathrm{d}}(10.1 \downarrow)$ \\
Hemi-cellulose & $25.0 \pm 0.3^{\mathrm{b}}$ & $24.6 \pm 0.1^{\mathrm{b}}(1.6 \downarrow)$ & $31.9 \pm 0.6^{\mathrm{a}}$ & $28.0 \pm 0.1^{\mathrm{c}}(12.2 \downarrow)$ \\
Lignin & $6.5 \pm 0.2^{\mathrm{a}}$ & $6.2 \pm 0.2^{\mathrm{a}}(4.6 \downarrow)$ & $5.0 \pm 0.4^{\mathrm{b}}$ & $4.5 \pm 0.2^{\mathrm{b}}(10.0 \downarrow)$ \\
Silica & $7.9 \pm 0.1^{\mathrm{a}}$ & $7.7 \pm 0.1^{\mathrm{a}}(2.5 \downarrow)$ & $4.2 \pm 0.2^{\mathrm{b}}$ & $3.9 \pm 0.2^{\mathrm{b}}(7.1 \downarrow)$ \\
\hline
\end{tabular}

*Experimental conditions; Control: Untreated paddy straw; values are means \pm standard deviation $(\mathrm{n}=3)$; Initial indicate sample taken after pretreatment; Final indicate sample taken after 45 days of pretreatment; value in parenthesis indicates \% increase $(\uparrow) ; \%$ decrease $(\downarrow)$ w.r.t initial value; Within the same row, different small letters indicate a significant difference between parameters under the different pretreatments based on Tukey's HSD test $(\mathrm{p}<0.05)$

Table 5.Change in proximate and chemical composition of microwave and biological pretreated paddy straw

\begin{tabular}{|c|c|c|c|c|c|c|}
\hline \multirow{2}{*}{$\begin{array}{l}\text { Composition of PS } \\
(\%)\end{array}$} & \multicolumn{2}{|c|}{ Control } & \multicolumn{2}{|c|}{$\begin{array}{l}\text { Microwave } \\
\text { (30 min) }\end{array}$} & \multicolumn{2}{|c|}{$\begin{array}{l}\text { Biological pretreatment with } \\
\text { Pleurotus ostreatus MTCC } 142\end{array}$} \\
\hline & Initial & Final & Initial & Final & Initial & Final \\
\hline Total solids & $96.2 \pm 0.4^{\mathrm{a}}$ & $95.4 \pm 0.7^{\mathrm{b}}(0.8 \downarrow)$ & $94.2 \pm 0.3^{\mathrm{c}}$ & $91.4 \pm 0.12^{\mathrm{d}}(2.9 \downarrow)$ & $93.8 \pm 0.2^{\mathrm{c}}$ & $90.2 \pm 0.1^{\mathrm{e}}(3.8 \downarrow)$ \\
\hline Volatile solids & $86.8 \pm 0.5^{\mathrm{a}}$ & $85.5 \pm 0.3^{\mathrm{b}}(1.4 \downarrow)$ & $79.4 \pm 0.1^{\mathrm{d}}$ & $73.2 \pm 0.2^{\mathrm{e}}(7.8 \downarrow)$ & $80.5 \pm 0.3^{\mathrm{c}}$ & $71.5 \pm 0.1^{\mathrm{f}}(11.1 \downarrow)$ \\
\hline Total organic carbon & $48.2 \pm 0.4^{\mathrm{a}}$ & $47.5 \pm 0.7^{\mathrm{b}}(1.4 \downarrow)$ & $44.1 \pm 0.2^{\mathrm{d}}$ & $40.6 \pm 0.1^{\mathrm{e}}(7.9 \downarrow)$ & $44.7 \pm 0.4^{\mathrm{c}}$ & $39.7 \pm 0.4^{\mathrm{f}}(11.1 \downarrow)$ \\
\hline Ash & $13.2 \pm 0.6^{\mathrm{e}}$ & $14.5 \pm 0.6^{\mathrm{d}}(9.8 \uparrow)$ & $20.6 \pm 0.1^{\mathrm{c}}$ & $24.8 \pm 0.5^{\mathrm{b}}(20.3 \uparrow)$ & $20.5 \pm 0.2^{\mathrm{c}}$ & $28.5 \pm 0.2^{\mathrm{a}}(39.0 \uparrow)$ \\
\hline cellulose & $46.2 \pm 0.5^{\mathrm{a}}$ & $45.8 \pm 0.2^{\mathrm{a}}(0.8 \downarrow)$ & $42.8 \pm 0.4^{b}$ & $38.6 \pm 0.4^{\mathrm{d}}(9.8 \downarrow)$ & $39.1 \pm 0.2^{\mathrm{c}}$ & $35.8 \pm 0.3^{\mathrm{e}}(8.4 \downarrow)$ \\
\hline Hemicellulose & $25.0 \pm 0.3^{\mathrm{a}}$ & $24.6 \pm 0.1^{\mathrm{a}}(1.6 \downarrow)$ & $22.2 \pm 0.3^{\mathrm{b}}$ & $20.3 \pm 0.3^{\mathrm{c}}(8.5 \downarrow)$ & $20.2 \pm 0.4^{\mathrm{c}}$ & $17.3 \pm 0.1^{\mathrm{d}}(14.3 \downarrow)$ \\
\hline Lignin & $6.5 \pm 0.2^{\mathrm{a}}$ & $6.2 \pm 0.2^{\mathrm{a}}(4.6 \downarrow)$ & $6.2 \pm 0.18^{\mathrm{a}}$ & $4.9 \pm 0.4^{\mathrm{b}}(20.9 \downarrow)$ & $6.0 \pm 0.4^{\mathrm{a}}$ & $5.2 \pm 0.4^{\mathrm{b}}(13.3 \downarrow)$ \\
\hline silica & $7.9 \pm 0.1^{\mathrm{a}}$ & $7.7 \pm 0.1^{\mathrm{a}}(2.5 \downarrow)$ & $6.4 \pm 0.2^{\mathrm{c}}$ & $4.4 \pm 0.4^{\mathrm{e}}(31.2 \downarrow)$ & $7.3 \pm 0.1^{\mathrm{b}}$ & $4.9 \pm 0.2^{\mathrm{d}}(32.8 \downarrow)$ \\
\hline
\end{tabular}

*Experimental conditions: \pm values indicate standard error of the triplicate data; Control: Untreated paddy straw; PS: Paddy straw; Initial indicate sample taken after pretreatment; Final indicates sample taken after 45 days of pretreatment; value in parenthesis indicates \% increase $(\uparrow)$; \% decrease $(\downarrow)$ w.r.t initial value; Within the same row, different small letters indicate a significant difference between parameters under the different pretreatments based on Tukey's HSD test $(\mathrm{p}<0.05)$ 
Table 6. Biogas production from different pretreatments of paddy straw

\begin{tabular}{|c|c|c|c|c|c|c|c|c|}
\hline \multirow{3}{*}{$\begin{array}{l}\text { Biogas } \\
\text { Production }\end{array}$} & \multicolumn{8}{|c|}{ Different Pretreatments } \\
\hline & \multirow[b]{2}{*}{ Control } & \multicolumn{2}{|c|}{$\mathrm{Na}_{2} \mathrm{CO}_{3}$} & \multicolumn{2}{|c|}{$\mathrm{NaOH}$} & \multirow{2}{*}{$\begin{array}{c}\text { Microwave } \\
\text { Pretreat- } \\
\text { ment } \\
(30 \text { min }) \\
\end{array}$} & \multirow{2}{*}{$\begin{array}{c}\text { Biological } \\
\text { Pretreat- } \\
\text { ment } \\
\text { (7 days) }\end{array}$} & \multirow{2}{*}{$\begin{array}{c}\text { Enzymatic } \\
\text { Pretreat- } \\
\text { ment } \\
(24 \mathrm{hrs})\end{array}$} \\
\hline & & $1 \%$ & $2 \%$ & $1 \%$ & $2 \%$ & & & \\
\hline $\begin{array}{l}\text { Biogas } \\
(\mathrm{ml} / 250 \mathrm{~g} \text { PS })\end{array}$ & $14.0 \pm 1.0^{\mathrm{h}}$ & $16.30 \pm 1.1^{\mathrm{g}}$ & $20.12 \pm 1.4^{\mathrm{b}}$ & $18.0 \pm 1.1^{\mathrm{e}}$ & $21.12 \pm 1.8^{\mathrm{a}}$ & $16.8 \pm 1.3^{f}$ & $20.0 \pm 0.9^{c}$ & $18.4 \pm 2.2^{\mathrm{d}}$ \\
\hline $\begin{array}{l}\text { Biogas }(\mathrm{L} / \mathrm{Kg} \\
\mathrm{PS})\end{array}$ & $56.1 \pm 4.0^{\mathrm{h}}$ & $\begin{array}{c}65.2 \pm 4.4^{\mathrm{g}} \\
(16.4 \uparrow)\end{array}$ & $\begin{array}{c}80.5 \pm 5.6^{\mathrm{b}} \\
(43.7 \uparrow) \\
\end{array}$ & $\begin{array}{c}72.0 \pm 4.4^{\mathrm{e}} \\
(28.5 \uparrow) \\
\end{array}$ & $\begin{array}{c}84.4 \pm 7.2^{\mathrm{a}} \\
(49.7 \uparrow) \\
\end{array}$ & $\begin{array}{c}67.2 \pm 5.2^{\mathrm{f}} \\
(19.7 \uparrow) \\
\end{array}$ & $\begin{array}{c}80.0 \pm 3.6^{\mathrm{c}} \\
(42.6 \uparrow) \\
\end{array}$ & $\begin{array}{c}73.6 \pm 8.8^{\mathrm{d}} \\
(31.1 \uparrow) \\
\end{array}$ \\
\hline
\end{tabular}

*Experimental conditions: PS: paddy straw; control: untreated paddy straw; \pm values indicate standard error of the triplicate data; value in parenthesis indicates \% increase $(\uparrow)$; \% decrease $(\downarrow)$ w.r.t control; Within the same row, different small letters indicate a significant difference between parameters under the different pretreatments based on Tukey's HSD test $(\mathrm{p}<0.05)$

hemicellulose by $11.8 \%$ was observed in $2 \%$ pretreated sample whereas lignin and silica were decreased by $16.0 \%$ and $12.1 \%$ respectively. The decrease in cellulose and hemicellulose is a clear cut indication of use of these components for biogas production by anaerobic microorganisms. Wang et al. (2001) studied proximate and chemical analysis of $P$. ostreatus impregnated spent grain samples at $20^{\circ} \mathrm{C}$ for 7 days and reported that spent grains consists of $14 \%$ cellulose, $9.1 \%$ lignin and $3.3 \%$ ash. Paddy straw rich in dry organic matter and cannot be consumed in one cycle. The proximate and chemical composition of residual paddy straw after biogas production indicate that there is still considerable amount of cellulose, hemicellulose and other nutrients which can be used for other applications like composting, vermi-composting etc.

Yang et al. (2013) observed 40.3\% delignification when straw was pretreated with $2 \%$ sodium carbonate whereas $9.6 \%$ delignification was reported by Khaleghian et al. (2015) when rice straw was pretreated with $1 \mathrm{M}$ sodium carbonate for $7 \mathrm{~h}$. However Kaur and Phutela, (2016) reported 32\% desilicification when paddy straw was pretreated with $2 \% \mathrm{Na}_{2} \mathrm{CO}_{3}$. Park and Kim, (2014) also showed 19\% delignification with sodium carbonate pretreatment of eucalyptus residues. However a higher proportion of lignin removal ranging from $39.5 \%$ to $72.5 \%$ was observed in $\mathrm{Na}_{2} \mathrm{CO}_{3}$ pretreated rice straw with temperature rising from $120^{\circ} \mathrm{C}$ to $150^{\circ} \mathrm{C}$ respectively (Yang et al., 2012).

Biogas production from $\mathrm{Na}_{2} \mathrm{CO}_{3}$ pretreated paddy straw: Table 6 presents the volumetric contents of methane in the reactor using rice straw as a substrate. The 2\% sodium carbonate pretreatment of paddy straw increased the biogas production by $43.7 \%$ from control. This may be due to lignin and silica reduction, however $1 \% \mathrm{Na}_{2} \mathrm{CO}_{3}$ pretreated sample produced only 65.2 litre biogas/Kg paddy straw with $16.4 \%$ increase. Kaur and phutela, (2016) reported $20.7 \%$ increase in biogas production when paddy straw was pretreated with $4 \% \mathrm{Na}_{2} \mathrm{CO}_{3}$. Dehghani et al. (2015) pretreated rice straw with $0.5 \mathrm{M}$ sodium carbonate at $110^{\circ} \mathrm{C}$ for 2 $\mathrm{h}$ which led to the improvement in biogas production due to significant reduction in the crystallinity of cellulose and lignin.

NaOH pretreatment: Maximum total solids were decreased in $2 \%$ pretreatment of paddy straw i.e. $1.7 \%$ and the decrease was significant $(\mathrm{p}<0.05)$ as shown in table 1 . Volatile solids also decreased significantly i.e. by $15.1 \%$ whereas $15.0 \%$ reduction was observed in total organic carbon content. The ash content was increased maximum by $45.4 \%$ in $2 \%$ pretreated sample. Cellulose and hemicellulose were consumed by 17.6 $\%$ and $23.2 \%$ in $2 \%$ pretreated sample respectively. Maximum amount of lignin reduced was $21.8 \%$ and silica was found to be increased by $20.0 \%$. The increase in silica may be due to the result of breakdown of silica in its free form. Van Soest, (2006) also reported that $\mathrm{NH}_{3}$ and urea cracks the silicified cuticular layer of rice straw but did not digest silica in contrast to the action of $\mathrm{NaOH}$. Similar results were obtained by Zhang and Cai, (2008) who found that $\mathrm{NaOH}$ pretreatment $(2 \%)$ for 1 hour at $85^{\circ} \mathrm{C}$ could reduce lignin and hemi-cellulose by $36.24 \%$ and $61.07 \%$ respectively. Wang et al., (2012) pretreated coastal Bermuda grass with of $0.5 \%$ to $3 \% \mathrm{NaOH}$ solution for a time period of 15 to $90 \mathrm{~min}$ which resulted in $86 \%$ lignin removal, however treatment of wheat straw with $1 \%$ $\mathrm{NaOH}$ for $1.5 \mathrm{hrs}$ decrease the hemicelluloses and lignin content by $44.15 \%$ and $42.52 \%$ respectively. However Harun and Goek, (2016) reported 79.0\% desilicification of sodium hydroxide $(12 \% \mathrm{w} / \mathrm{v})$ pretreated paddy straw. He et al. (2008) showed that rice straw pretreated with $6 \% \mathrm{NaOH}$ solution reduced cellulose, hemicellulose, and total lignocellulose by $88.0 \%, 75.0 \%$, and $72.3 \%$ respectively. A close observation was made my Jaisamut et al. (2013) who reported $59.7 \%$ lignin removal from $0.05 \mathrm{~g}$ of $\mathrm{NaOH}$ pretreatment of wheat straw at $140^{\circ} \mathrm{C}$ for $85 \mathrm{~min}$. Cheng et al. (2010) also showed delignification rate from $8.6 \%$ to $23.1 \%$ respectively. Similar results were observed when Omidvar et al. (2016) pretreated rice straw with $2.6 \mathrm{M} \mathrm{NaOH}$ at $80^{\circ} \mathrm{C}$ for 150 min resulting in $31.3 \%$ delignification.

Decrease in total solids might be due to the loss of some of the components of paddy straw as a result of their solubilisation. The decrease in cellulose was the 
clear-cut indication of the breakdown of lignin-hemicellulose complex thereby resulting in a greater loss in the hemi-cellulose and lignin content while silica reduction might due to the fact that $\mathrm{NaOH}$ solubilized silica, converting silica to its monomeric forms thus releasing free silicic acids.

Biogas production from $\mathrm{NaOH}$ pretreated paddy straw: The hydrolysis of $\mathrm{NaOH}$ pretreated rice straw showed a $49.7 \%$ hike in biogas production with increase in $\mathrm{NaOH}$ concentration from $1 \%$ to $2 \%$ (table 6). A maximum of 84.4 litre biogas $/ \mathrm{kg}$ paddy straw was obtained in case of $2 \% \mathrm{NaOH}$ concentration, whereas in untreated paddy straw only 56.1 litre biogas $/ \mathrm{kg}$ paddy straw was produced. Similar results were obtained by Dai et al. (2014) in which they found that rice straw pretreated with $4 \%, 6 \%$ and $8 \% \mathrm{NaOH}$ solutions gave a total biogas production of $12.1,18.7$ and 15.0 litre respectively. A total of $64.5 \%$ increase in biogas production was observed by $\mathrm{He}$ et al. (2009) when rice straw was pretreated with $6 \% \mathrm{NaOH}$ solution. Similarly Qiu et al. (2011) pretreated peanut shells with $4 \%$ and $8 \%$ sodium hydroxide solutions which led to 28.0 litre and 17.6 litre biogas production. Silicase production in submerged fermentation: Silicase production from P.ostreatus MTCC 142 was studied in submerged fermentation for a period of 10 days in silica peptone broth and results are depicted in table 2. With the increase of incubation period, the enzyme activity increased. The maximum silicase activity was found to be $1.05 \mathrm{U} / \mathrm{ml}$ on $8^{\text {th }}$ day of incubation and a reduction in enzyme activity was observed after $8^{\text {th }}$ day, this might be due to the fact that peptone which acts as nitrogen source is consumed by $8^{\text {th }}$ day for carrying out metabolic activities for the growth of the microorganism. Report on silicase production by Pleurotus ostreatus is not available however, Negi and Kumari, (2014) observed maximum laccase activity of $210 \mathrm{U} / \mathrm{g}$ produced from P.ostreatus MTCC 1802 at $25^{\circ} \mathrm{C}$ on $7^{\text {th }}$ day of incubation by using wheat bran and neem bark as substrate. Similar trend was also observed for carbonic anhydrase (silica solubilzation) activity (Schroder et al., 2003) which was marginally higher as compared to silicase.

Silica is especially observed in outer epidermal walls and possesses a complex chemical structure which makes it resistant to enzymatic attacks. Armstrong et al. (1966) demonstrated that silicase also exhibits carbonic anhydrase activity and can be measured by the colorimetric assay.

Wang et al. (2010) reported that silicase is a member of the family of carbonic anhydrases. Comparison of sponge silicase sequence with human carbonic anhydrase (II) shows that most of amino acid characteristics to CA's are also present in sponge silicase. As carbonic anhydrase catalyses very fast reversible reaction, so further studies were conducted by determining the silicase activity by the method of Toender and Borchert, (2014).

Enzymatic hydrolysis
Pretreatment with crude silicase: Chopped and soaked paddy straw was pretreated with crude enzyme extract with different enzyme concentrations i.e. 15, 30 and 45 units $/ 10 \mathrm{~g}$ of paddy straw for $24 \mathrm{hrs}$. A significant increase in total solids was noticed. The volatile solids and total organic carbon were reduced by $6.6 \%$ and $6.6 \%$ respectively (table 3 ). The ash content drastically increased in all cases but maximum increase was found to be $43.9 \%$ with $45 \mathrm{U}$ silicase $/ 10 \mathrm{~g}$ paddy straw in the treated sample, which may be due to the decrease in volatile solids. Cellulose decreased maximally by $16.8 \%$ but hemicellulose was found to increase by $27.6 \%$ with $45 \mathrm{U}$ silicase/10 g paddy straw. However lignin and silica showed maximum reduction i.e. $23.0 \%$ and $46.8 \%$ respectively. This large change in silica demonstrates that P.ostreatus MTCC 142 is silica degrading fungi and can improve biogas production. Our results are in corroboration with that of Kaur, (2013) with $45 \%$ silica reduction in paddy straw in P.ostreatus MTCC 142 impregnated rice straw at $28^{\circ}$ $\pm 2^{\circ} \mathrm{C}$ for a period of 8 days.

Biogas production from paddy straw pretreated with crude silicase: Pretreatment of paddy straw with crude silicase (45 U/g PS) resulted in the increase in biogas production by $31.1 \%$ as compared to control which produced only 56.1 litre biogas $/ \mathrm{Kg}$ paddy straw (table 6). Kaur, (2009) also showed 15\% increase in biogas production from paddy straw pretreated with $P$. florida on $15^{\text {th }}$ day of incubation.

Proximate and chemical composition of silicase pretreated spent paddy straw: Asignificant change $(p<0.05)$ was observed in the total solid content, whereas volatile solids and total organic carbon reduced by $4.6 \%$ and $4.8 \%$ respectively as depicted in table 4 . This may be due to the fact that organic fraction of paddy straw is utilized for biogas production. Ash content increased by $20.0 \%$ whereas cellulose and hemicellulose content were reduced by $10.1 \%$ and $12.2 \%$ respectively. The lignin and silica reduced by $10.0 \%$ and $7.1 \%$ respectively.Similar results with a $19.1 \%$ lignin reduction were observed in Coriolus versicolor MTCC 138 pretreated paddy straw after 25 days of incubation (Phutela and Sahni, 2013).

Microwave and biological pretreatment of paddy straw: In order to investigate the different pretreatment methods the straw was irradiated with microwaves and biological pretreatment. The physical, structural as well as the chemical properties were changed during the microwave pretreatment, as discussed in table 5 . There was a significant $(\mathrm{p}<0.05)$ loss in total solids volatile solids and total organic carbon content with 2.9 $\%, 7.8 \%$ and $7.9 \%$ after pretreatment, but the ash content was enhanced by $20.3 \%$ which may be due the reduction in volatile solids. The respective decomposition rates of cellulose and hemicellulose were $9.8 \%$ and $8.5 \%$ respectively. Higher amount of lignin and silica was dissolved i.e. $20.9 \%$ and $31.2 \%$ which may be due to the reaction caused by radiation, but the entire mechanism is still unclear. Zhu et al. (2005) reported cellulose reduction by $69.2 \%$, lignin $4.9 \%$ and hem- 
icellulose $10.2 \%$ after 30 -min microwave/alkali pretreatment. Similarly Akhtar et al. (2014) showed 39\% lignin reduction with assisted microwave-acid pretreatment of leaf litter.

All the above mentioned methods like chemical and microwave pretreatments areharsh and cost intensive, therefore biological degradation of biomass is preferred now days which is not only cost prone but also enlist eco-friendly behaviour. The method mainly involves the use of actinomycetes, bacteria, white and soft rot-fungi which corrupts the lignin structure, a highly complex polymer present in biomass. The total solid and volatile solid content were reduced by $3.8 \%$ and $11.1 \%$ in biological pretreatment while the ash content was increased by $39.0 \%$. There was a profound loss in cellulose and hemicellulose content with $8.4 \%$ and $14.3 \%$ respectively. The high loss in hemicellulose might due to the fact that wood-decaying fungi are able to metabolize and decompose all plant cell constituents (lignin, hemicellulose and cellulose) by their enzymes. Lignin and silica also found to be minimized by $13.3 \%$ and $32.8 \%$ respectively. Some white-rot fungi are able to decompose free phenolic monomers and break the bonds with which lignin is cross-linked to the polysaccharides in rice straw (Chen et al., 1996) which makes it more susceptible to microbial attack. The results were in corroboration with Phutela and Sahni, (2013) who found that paddy straw pretreated with Coriolus versicolor MTCC 138 decreased cellulose by $19.3 \%$ whereas lignin and silica by $19.1 \%$ and $32.5 \%$, respectively. Thakur et al. (2013) also showed that pretreatment of wheat straw and banana straw with P.ostreatus HP-1which led to the removal of $49.2 \%$ lignin, $12.4 \%$ cellulose and $21.8 \%$ hemicelluloses. Many other researchers also focused on paddy straw pretreatment using fungal mechanisms. Mustafa et al. (2016) pretreated rice straw with P.ostreatus (DSM 11191) which led to $33.4 \%$ lignin removal. Similar results were reported by Balasubramanium and Rajarathinum, (2013) who showed $27.8 \%$ and $24.4 \%$ lignin and cellulose reduction respectively by P.ostreatus pretreatment of paddy straw.

Biogas production from Microwave and biological pretreated paddy straw: A summary of results from the biogas potential approach of the microwave pretreated as well as the fungal pretreated straw with respect to the controlis shown in table 6. A total of 67.2 litre biogas $/ \mathrm{Kg}$ of paddy straw was produced in the microwave irradiated sample corresponding to the biological pretreatment method which produced 80.0 liters biogas $/ \mathrm{Kg}$ paddy straw. Both pretreatments showed $19.7 \%$ and $42.6 \%$ increase in biogas production with respect to control. There is possibility of conversion of lignin into other soluble components which may further be utilized by the methanogens as a substrate after the consumption of simpler/preferred substrates i.e. cellulose/hemicellulose. The result justified that biological pretreatment could enhance the methane yield by removing more cellulose, hemicellulose and silica content from paddy straw as compared to microwave treated and untreated paddy straw.

\section{Conclusion}

Lignin and silica reduction significantly affected the biogas production in chemically pretreated straw with $43.7 \%$ increase in $\mathrm{Na}_{2} \mathrm{CO}_{3}$ and $49.7 \%$ in $\mathrm{NaOH}$ with respect to control. However crude enzymatic pretreatment enhanced biogas yield by $31.1 \%$ with $23.0 \%$ and $46.8 \%$ decrease in lignin and silica content. For $P$. ostreatus pretreatment lignin and silica removal was found to be $13.3 \%$ and $32.8 \%$ at 7 days of incubation time resulting in $42.6 \%$ increase in methane level. Both chemical and fungal pretreatments showed a linear interrelationship with similar values for biogas production but weakly related to silica degradation during chemical pretreatment. It was concluded that cellulose and hemicellulose breakdown in higher proportion leads to less degradation of lignin and silica content in $\mathrm{NaOH}$ pretreated paddy straw. Although chemical pretreatment can efficiently increase biogas production but can be detrimental to the environment, therefore fungal pretreatment is preferred for digestibility of rice straw and biogas yield.

\section{ACKNOWLEDGEMENTS}

The authors acknowledge the financial support provided by AICRP-BCT (All India Coordinated Research Project, Bioconversion Technology) ICAR (Indian Council of Agricultural Research) for pursuing this project.

\section{REFERENCES}

Ai, B., Chi, X., Meng, J., Sheng, Z., Zheng, L., Zheng, X. and Li, J. (2016). Consolidated Bioprocessing for Butyric Acid Production from Rice Straw with Undefined Mixed Culture. Front Microbiol. 7 :1-10.

Akhtar, N., Jain, A. K., Goyal, D. and Goyal, A. (2014). Surfactant assisted microwave-acid pretreatment of leaf litter biomass for enhanced enzymatic release of sugars. Proc 7th International Conference on Environmental Science and Technology Department of Biotechnology, Thapar University, Patiala, 147004, Punjab, India

Akhter, S., Kotru, R., Dar, N. A., Rasool, R. and Din, R. M. U. (2015). Resource conservation technology in rice wheat cropping system. Best: JHAMS. 1 (2) : 25-28

AOAC (2000) Association of Official Analytical Chemists, Official methods of Analysis, J AOAC Int. 83 (4): 1020$25,17^{\text {th }}$ Edition, Maryland, USA

Armstrong, J. M., Myers, D. V., Verpoorte, J. A. and Edsall, J. T. (1966). Purification and properties of human erythrocyte carbonic anhydrase. J. Biol Chem. 241 : 51374519

Arvanitoyannis, I. S. and Tserkezou, P. (2008). Corn and rice waste: A comparative and critical presentation of methods and current and potential uses of treated waste. Int J Food Sci. Tech., 43 : 958-988 
Balasubramaniam, M. K. and Rajarathinam, R. (2013). Implementation of White rot fungal Pretreated Rice straw for Sustainable Bioethanol Production by Saccharomyces cerevisiae. IJERT, 2 (11) : 4047-4053

Blanchette, R. A., Burnes, T. A., Eerdmans, M. M. and Akhtar, M. (1992). Evaluating isolates of Phanerochaete chrysosporium and Ceriporiopsis subvermispora for use in biological pulping purposes. Holzforschung. 42 : $109-115$

Chen, J., Fales, S. L., Varga, G. A. and Royse, D. J. (1996). Biodegradability of free monomeric cell-wall-bound phenolic acids in maize stover by two strains of whiterot fungi.J. Sci. Food Agr., $71:$ 145-150

Cheng, S. Y.,Zheng, Y., Yu, C. W .,Zheng, Y., Yu, C. W., Dooley, T. M., Jenkins, B. M., Jean, S. and Gheynst, V .(2010). Evaluation of High Solids Alkaline Pretreatment of Rice Straw. Appl. Biochem Biotechnol., 162 : $1768-1784$

Contreras, L. M., Schelle, H., Sebrango, C. R. and Pereda, I. (2012). Methane potential and biodegradability of rice straw, rice husk and rice residues from the drying process. Wat Sci Tech., 65 (6) : 1142-1149

Dai, B., Zhu, A., Mu, F., Xu, N. and Wu, Z. (2014). Experiments on anaerobic digestion of rice straw for biogas production under $\mathrm{NaOH}$ pretreatment. Adv Mat Res., 953-954 : 220-223

Dehghani, M., Karimi, K. and Sadeghi, M. (2015). Pretreatment of rice straw for the improvement of biogas production. Energ Fuels. 29 : 3770-3775

Economic uses of rice straw, Cultural rice research board http://www.carrb.com/84rpt/StrawUses.htm, accessed online: $18 / 11 / 16$

FAO and Rice Market Monitor (2014). Trade and Markets Division Food and Agriculture Organization of the United Nations. 17: 4

Gadde, B., Bonnet, S., Menke, C. and Garivait, S. (2009). Air pollutant emissions from rice straw open field burning in India, Thailand and the Philippines. Environ Pollut., 157 : 1554-1558

Gammal, A., Kamel, Z., Adeeb, Z. and Helmy, S. M. (1998). Biodegradation of lignocellulosic substances and production of sugars and lignin degradation intermediates by four selected microbial strains. Polym.Degrad Stab., $61: 535-542$

Harun, S. and Goek, S. K. (2016). Effect of Sodium Hydroxide Pretreatment on Rice Straw Composition. Indian $J$ Sci Technol., 9 (21): 1-9

He, Y., Pang, Y., Liu, Y., Li, X. and Wang, K. (2009). Physico chemical characterization of rice straw pretreated with sodium hydroxide in the solid state for enhancing biogas production. Energ. Fuels, 22 : 2775-2781

Henderson, M. E. K. and Duff, R. B. (1965). The release of metallic and silicate ions from mineral rocks and soils by fungal activity. J. Soil Sci., $14: 236-246$

Jafri, U. A., Javed, M. T. and Chugtai, I. R. (2011). Process investigation for conversion of municipal solid waste into liquid fuel. JPIChE. $39: 23-27$

Jaisamut K., Paulová L., Patáková P., Rychtera M. and Melzoch, M. (2013). Optimization of alkali pretreatment of wheat straw to be used as substrate for biofuels production. Plant Soil Environ., 59 (12) : 537-542

Kangle, K. M., Kore, S. V., Kore, V. S. and Kulkarni, G. S. (2012). Recent trends in anaerobic co-digestion: A Re- view. UJERT. 2 (4) : 210-219

Kaur, K. (2009). Effect of chemicals and biological treatment of paddy straw on biogas production. M.Sc Thesis, Punjab Agricultural Univeristy, Ludhiana

Kaur, K. (2013). Development of lignin and silica solubilizing microbial consortium for paddy straw pretreatment to enhance biogas production Ph.D. dissertation, Punjab Agricultural Univeristy, Ludhiana

Kaur, K. and Phutela, U. G. (2016). Sodium carbonate pretreatment: an approach towards desilicification of paddy straw and enhancement in biogas production. Paddy Water Environ., 14 (1) : 133-221

Khaleghian, H., Karimi, K. and Behzad, T. (2015). Ethanol production from rice straw by sodium carbonate pretreatment and Mucorhiemalis fermentation. Ind Crop Prod., 76 : 1079-1085

Kumar, P., Barrett, D. M., Delwiche, M. J. and Stroeve, P. (2009). Methods for pretreatment of lignocellulosic biomass for efficient hydrolysis and biofuel production. Ind Eng Chem Res., 48 : 3713-3729

Mustafa, A. M., Poulsen, T. G., and Sheng, K. (2016). Fungal pretreatment of rice straw with Pleurotusostreatus and Trichoderma reesei to enhance methane production under solid-state anaerobic digestion. Appl. Energy, $180: 661-671$

Negi, S. and Kumari, J. (2014). Development of bioprocess for the production of laccase by Pleurotus ostreatus MTCC 1802 using evolutionary optimization technique. Indian J Exp Biol., 52 (11) : 1106-1111

Omidvar, M., Karimi, K. and Mohammadi, M. (2016). Enhanced ethanol and glucosamine production from rice husk by $\mathrm{NAOH}$ pretreatment and fermentation by fungus Mucorhiemalis. Biofuel Res J. 11 : 475-481

Park, C. Y. and Kim, S. J. (2014). Comparison of various alkaline pretreatment methods of lignocellulosic biomass. Energy., 47 (1) :31-35

Phutela, U. G. and Sahni, N. (2013). Microscopic structural changes in paddy straw pretreated with Trichoderma reesei MTCC 164 and Coriolus versicolor MTCC 138 Indian J. Microbiol., 53 (2) : 227-231

Platt, M. W, Hadar, Y. and Chet, I. (1984). Fungal activities involved in lignocellulose degradtion by Pleurotus spp. Appl. Microbiol. Biotechnol. 20 (2): 150-154

Qiu, Y. S., Ling, Q., Yong, S., Tao, L. and Fang, Y. D. (2011). Experiments on anaerobic digestion of $\mathrm{NaOH}$ pretreated peanut shell for biogas production. J. AgroEnviron Sci., 30 (3) : 573-578

Raeisi, S. M., Tabatabaei, M., Ayati, B., Ghafari, A. and Mood, S. H. (2016). A novel combined pretreatment method for rice straw using optimized EMIM [Ac] and Mild NaOH. Waste Biomass Valor., 7 : 97-107

Renewables Global Status Report (2016). Renewable Energy Policy Network for the $21^{\text {st }}$ Century (REN 21), www.ren21.net.ISBN 978-3-98181-0-7

Saha, B. C. (2003). Hemicellulose bioconversion. J Ind Microbiol Biotechnol., 30 : 279-291

Saini, J. K. and Saini, R. (2015). Lignocellulosic agriculture wastes as biomass feedstocks for second-generation bioethanol production: concepts and recent developments. Biotechnol., 5 : 337-353

Schroder, H. C., Krasko, A., Pennec, G. L. and Muller, W. E. G. (2003). Silicase, an enzyme which degrades biogenous amorphous silica: Contribution to the metabolism 
of silica deposition in the demosponge Suberites domuncula. Prog Mol Subcell Biol., 33 : 249-268

Soest, V. (2006). Rice straw, the role of silica and treatments to improve quality. Anim Feed Sci Technol., 130 (3-4) : 137-171

Streets, D. G., Yarber, K. F., Woo, J. H. and Carmichael, G. R. (2003). Biomass burning in Asia: Annual and seasonal estimates and atmospheric emissions. Glob. Biogeochem Cycles 17 : 1099-1119

Taniguchi, M., Suzuki, H., Watanabe, D., Sakai, K., Hoshino, K. and Tanaka, T. (2005). Evaluation of pretreatment with Pleurotus ostreatus for enzymatic hydrolysis of rice straw. J Biosci Bioeng., 100 : 637-643

Thakur, S., Shrivastava, B., Ingale, S., Kuhad, R. C. and Gupte, A. (2013). Degradation and selective ligninolysis of wheat straw and banana stem for an efficient bioethanol production using fungal and chemical pretreatment. Biotechnol., 3 (5) : 365-372

Toender, E. J. and Borchert, T. M. (2014). Use of enzymes having silicase activity US20140335577A1

Wang, D., Sakoda, A. and Suzuki, M. (2001). Biological efficiency and nutritional value of Pleurotus ostreatus cultivated on spent beer grain. Bioresource Technol., 78:293-300

Wang, X., Hu, S., Gan, L., Wiens, M. and Muller, W. E. G. (2010). Sponges (Porifera) as living metazoan witnesses from the Neoproterozoic: biomineralization and the concept of their evolutionary success. Terra Nova., 22 (1) : 1-11

Wang, Y., Peng, Z., Liao, Q. and Chen, R. (2012). Effect of magnesium ions on biogas production characteristics of lettuce and potato peelings in anaerobic digester. $T$ Chi Soc of Agric Eng., 28 (6) : 217-221

Wang, Z., Keshwani, D. R., Redding, A. P.and Cheng, J. J. (2010). Sodium hydroxide pretreatment and enzymatic hydrolysis of coastal Bermuda grass. Bioresource Technol., 101 (10) : 3583-3585

Werle, L. B., Garcia, J. C., Kuhn, R. C., Schwaab, M., Foletto, E. L, Cancelier, A., Sergio, L. J. and Mazutti, M. A. (2013). Ultrasound-assisted acid hydrolysis of palm leaves (Roystonea oleracea) for production of fermentable sugars. Ind. Crops Prod. 45 : 128-132

Yang, L., Cao, J., Jin, Y., Chang,H. M., Jameel, H., Phillips, R. and Li, Z. (2012). Effects of sodium carbonate pretreatment on the chemical compositions and enzymatic saccharification of rice straw. Bioresource Technol. $124: 284-291$

Yang, L., Cao,J.,Mao, J. and Jin, Y. (2013). Sodium carbonate-sodium sulphite pretreatment for improving the enzymatic hydrolysis of rice straw. Ind Crop Product., $43: 711-717$

Younas, R., Zhang, S., Zhang, L., Luo, G., Chen, K., Cao, L., Liu, Y. and Hao, S. (2016). Lactic acid production from rice straw in alkaline hydrothermal conditions in presence of NiOnanoplates. Catal. Today., 274: 40-48

Zhang, Q. and Cai, W. (2008). Enzymatic hydrolysis of alkali pretreated rice straw by Trichoderma reesei ZM4-F3. Biomass Bioeng., 32 : 1130-1135

Zhu, S., Wu, Y., Yu, Z., Liao, J. and Zhang, Y. (2005). Pretreatment by microwave/alkali of rice straw and its enzymatic hydrolysis. Process Biochem., 40: 3082-3088 